authorized to work out the constitutional and other details of the scheme which would, in due course, be placed before the Council for consideration. The war has inevitably interrupted these consultations, but they will be resumed as soon as the international situation permits.

\title{
L'Art Congolais au Service du Culte.
}

Sous ce titre nous avons esquissé dans le numéro d'avril 1937 une doctrine de l'adaptation visant à se servir, dans l'œuvre de l'évangélisation en Afrique, de toutes les ressources que l'art indigène peut offrir. Nos lecteurs nous sauront gré, croyons-nous, d'être informés de deux essais intéressants, faits récemment en ce sens au Congo belge, en des domaines aussi variés que la musique et le dessin.

Le premier essai est dû au R.P. A. Walschap. ${ }^{I}$ Dès son arrivée en Afrique, il s'est efforcé à comprendre la musique nègre. Il remarqua bientôt que la mélodie n'en est qu'un élément secondaire, l'élément spécifique et distinct étant le rythme. Pour saisir celui-ci il faut remonter à la danse: aux battements syncopés des pieds, aux balancements des corps, aux mouvements élégants des torses et des bras, aux lentes inclinaisons et aux brusques secousses des têtes. Un temps principal donne la mesure, dirige les mouvements des corps ainsi que l'action des instruments musicaux; il les régit, leur permet des évolutions arbitraires mais sans qu'ils s'affranchissent de lui, toujours dominés par lui. Cette diversité dans l'unité est un pendant de la polyphonie classique.

L'auteur ne doute plus de la possibilité d'utiliser la musique indigène: on l'a introduite dans l'église. Lui-même composa en style indigène une messe et une ample série de motets. Ce n'est plus l'harmonium qui accompagne pareilles réalisations musicales, mais un orchestre d'instruments indigènes tels que des tambours, des sortes de hochets, des clochettes, etc. Il estime toutefois qu'il faut faire davantage: on doit aussi utiliser la danse. Celle-ci fait partie intégrante de la vie indigène: il doit en rester ainsi pour le nègre devenu chrétien. C'est pourquoi l'auteur a conçu le plan de composer une série de danses religieuses correspondant aux grandes fêtes de l'année liturgique. Déjà il a illustré dans un ensemble de manifestations chorégraphiques et musicales les évènements qui conduisent à la naissance du Christ (Noël) et à la résurrection (Pâques). Ces danses sont exécutées sur le parvis de l'église et connaissent, auprès des indigènes, le plus grand succès. Elles font penser directement aux Mystères du moyen-âge et constituent, comme telles, un début du théâtre indigène.

Le second essai, celui du R.P. Vandenhoudt, ${ }^{2}$ se rapporte au dessin. Quand, il y a quelques années, il fut envoyé comme missionnaire dans le

I 'Gedachten over Negermuziek', Aequatoria, mars 1939, $25-9$.

${ }^{2}$ Inlandsche Kunst. Missions de Scheut, mars 1939, 85-8. 
Bas-Congo, sa curiosité artistique l'amena à l'étude de l'art et des conceptions artistiques des indigènes et il chercha comment les dessins schématiques dont se servent les noirs pour présenter graphiquement leurs scènes de la vie, pourraient l'aider à faire des dessins adaptés à leur mentalité. Se fondant sur des impressions personnelles, il adapta leurs dessins graphiques à des représentations chrétiennes. Il fit des essais et se forma petit à petit un style spécial, qu'il mit au service de l'illustration de la Bible. Le résultat donne une interprétation de la Bible dans un esprit purement africain. Que les images ainsi réalisés soient claires pour l'intelligence des noirs, cela ressort du fait que les indigènes eux-mêmes lui expliquaient facilement la signification de chacune de ses gravures.

Très caractéristique pour son travail est par exemple le dessin représentant 'Le sacrifice d'Abraham'. Dans quelques régions d'Afrique, on liait la tête des condamnés à mort aux branches d'un arbre, pour que la tête coupée fût projetée en l'air. Cette technique le P. Vandenhoudt l'a transposée dans le sacrifice d'Abraham. Dans une mise en page magistrale et impressionnante l'arbre s'arque tiraillant le corps martyrisé de l'enfant, pendant que l'ange, d'un mouvement dramatique, arrête la main sur le point de frapper. C'est bien le texte, 'Ne porte pas la main sur l'enfant et ne lui fais rien' (Gen. xxii, 12) vivant devant les yeux. Dans le même cadre africain il dessine 'Joseph conduit en prison ': un soldat emmène un noir lié, scène connue de tous les indigènes. Appliquant le même principe, il représente 'Mö̈se brisant le veau d'or', 'L'adoration des bergers', ' Jésus, divin ami des enfants', 'La résurrection du fils de la veuve de Naïm', 'Adam donnant aux animaux leur nom', 'Le pauvre homme Job', etc.

Le R.P. Vandenhoudt a fait, comme on le voit, un essai d'adaptation pratique qui mérite toute l'attention. (Communication du Professeur N. DE Claekne.)

In connexion with the above note, our readers will be particularly interested to hear that, following on the steps taken by the Governments of the various British West African Colonies at the suggestion of the Secretary of State for the Protection of Indigenous Arts, Treasures and Monuments, similar steps have been taken in the Belgian Congo. The Bulletin Officiel du Congo Belge of 15 th September reproduces a decree signed by the King on the 16th August, 1939, "to ensure the protection of sites, monuments and productions of indigenous art'.

\section{Intelligence Tests applied to Negroes.}

THE testing of intelligence is a technique applied in experimental psychology. It is largely used by American psychologists and has led to remarkable results. In North America with its population composed of many races, 